\title{
Sonography of Wrist Ganglion Cysts Which Location Is Most Common?
}

\author{
Andrew Zhang, MD, Anna L. Falkowski, MD, MHBA, Jon A. Jacobson, MD (D, Sung Moon Kim, MD, \\ Sung Hye Koh, MD, Kara Gaetke-Udager, MD
}

Received October 23, 2018, from the Department of Radiology, University of Michigan, Ann Arbor, Michigan, USA (A.Z., A.L.F., J.A.J., S.M.K., S.H.K., K.G.-U.); Department of Radiology, University Hospital Basel, University of Basel, Basel, Switzerland (A.L.F.); and Department of Radiology, Hallym Sacred Heart Hospital, Hallym University, College of Medicine, Gyeonggi-do, South Korea (S.H.K.). Manuscript accepted for publication November 16, 2018.

A preliminary version of this research was presented at the American Institute of Ultrasound in Medicine's Annual Convention, March 2018.

Anna L. Falkowski, MD, MHBA, received grants from the Research Funds of the University of Basel, Swiss Society of Radiology, Gottfried und Julia BangerterRhyner-Stiftung, and Freiwillige Akademische Gesellschaft Basel. The sponsors played no role in matters of study design, collection, analysis, or interpretation of data or in the decision to submit the paper for publication.

Address correspondence to Jon A. Jacobson, MD, Department of Radiology, University of Michigan, 1500 East Medical Center Drive TC2910L, Ann Arbor, Michigan 48109-0326 USA.

E-mail: jjacobsn@umich.edu

doi:10.1002/jum.14912
Objectives-The literature states that wrist ganglion cysts are most commonly dorsal; however, our experience suggests a volar location is more common. The purpose of this study was to identify the locations of ganglion cysts of the wrist as imaged with sonography.

Methods-After Institutional Review Board approval with informed consent waived, retrospective review of wrist sonography reports from January to April 2016 was completed. Only patients who had a comprehensive evaluation that included the dorsal and volar wrist were included. Ultrasound images were reviewed to characterize wrist ganglion cysts.

Results - The study group consisted of 98 subjects ( $78 \%$ female, $22 \%$ male) (median age, 51 years; range 13-79) with 124 wrist ganglion cysts, where 69\% (86 of 124) were volar and 31\% (38 of 124) were dorsal. Ganglion cysts were located between the radial artery and flexor carpi radialis in 63\% (78 of 124), followed by a dorsal location superficial to the scapholunate ligament in $20 \%$ (25 of 124), other dorsal locations in 11\% (13 of 124), and other volar locations in $6 \%(8$ of 124). With dorsal ganglion cysts, the scapholunate ligament when imaged appeared normal in $91 \%$ (20 of 22). Ganglion cysts were multilocular/ multilobular in all subjects.

Conclusions - The most common location for wrist ganglion cysts is in the volar wrist, particularly between the radial artery and flexor carpi radialis tendon, appearing multilocular or multilobular with a mean largest dimension of $1.0 \mathrm{~cm}$. Given the propensity of ganglion cysts to occur at this site, attention to this area when imaging the wrist with sonography or magnetic resonance imaging should be considered.

Key Words - cyst; ganglion; UltrasoundWrist

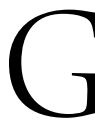
anglion cysts are benign mucin-filled cysts typically located near joints and tendons and are the most common soft tissue masses of the wrist. ${ }^{1,2}$ Although their etiology is unknown, theories include joint stress leading to leakage of fluid through a joint capsule, mucoid degeneration of periarticular connective tissue, or stimulation of mucin secretion by periarticular mesenchymal cells. ${ }^{2,3}$ Ganglion cysts commonly present with pain, tenderness, and interference of activity. ${ }^{1,2}$ Accurate diagnosis is important both to differentiate ganglion cysts from other pathologic conditions and to identify such cysts, as they can be effectively treated with ultrasound-guided aspiration. ${ }^{4}$

Sonography is commonly used to accurately detect wrist ganglion cysts. ${ }^{5,6}$ Wrist ganglia most commonly appear well-defined, multilobular or multilocular, noncompressible, and hypoechoic on sonography, measuring between 7 and $30 \mathrm{~mm} .^{5,6}$ Common sites 
for wrist ganglion cysts include the dorsal wrist superficial to the scapholunate ligament and the volar wrist between the radial artery and flexor carpi radialis tendon. ${ }^{2}$ While one limited study describes a volar location as more common, the majority of the literature states that ganglion cysts of the wrist are most commonly located dorsally, with $60 \%$ to $70 \%$ of ganglion cysts in this location. $2,3,5,7$

In our clinical practice, sonography is routinely used to assess for hand and wrist pathology, including ganglion cysts. We have anecdotally observed that a volar location may be more common than the current literature states. The purpose of this study was to retrospectively review the ultrasound locations of wrist ganglion cysts to clarify their most common locations.

\section{Materials and Methods}

The Institutional Review Board approved our study, and informed consent was waived. A retrospective search was completed for all wrist extremity ultrasound reports performed at our institution from January 2016 to April 2016. Wrist ultrasound reports that did not include a comprehensive evaluation of the dorsal and volar wrist were excluded. Ultrasound reports were then reviewed by one author to determine if a ganglion cyst was described, and then location and size of the ganglion cyst was recorded.

Ultrasound examinations of the wrist were performed by an ultrasound technologist supervised by one of 11 fellowship-trained musculoskeletal radiologists (experience ranged from 8 to 18 years) as part of routine clinical care. High-frequency probes $(12-18 \mathrm{MHz}$ ) were used (Philips, Model Epiq, Bothell, WA; GE, Model E9, Milwaukee, WI). The criteria used to diagnose a ganglion cyst was that of a welldefined, anechoic or hypoechoic, unilocular or multilocular/multilobular fluid collection not representing an anatomic bursa or joint recess, as described in the literature. ${ }^{6,8}$

In subjects with reported ganglion cysts, static ultrasound images and cine clips were then retrospectively reviewed on an imaging workstation in consensus by 2 authors to confirm the presence of a ganglion cyst, to ensure that the size and location of the ganglion cysts were accurately recorded, to record the locularity of the ganglion cyst (unilocular or multilocular/multilobular), to record blood flow on color Doppler imaging (presence or absence), and to assess the appearance of the dorsal component of the scapholunate ligament if a dorsal wrist ganglion was present. A scapholunate ligament was deemed to be normal if appeared hyperechoic and fibrillar in the $\mathrm{V}$-shaped articulation between the lunate and scaphoid in the axial plane, while the absence of a normal ligament was classified as abnormal. ${ }^{9}$ Subject demographics were also recorded. The term multilobular refers to the lobularity of the outer border of the cyst, while the term multilocular refers to the internal separate rounded areas separated by thin walls or septae, and are used synonymously for this research given their sonographic similarities at retrospective review. Patient medical records were reviewed with regard to indication for ultrasound examination, surgical data, and other imaging. Any patient with a history of prior wrist surgery or prior treatment of a ganglion cyst was excluded.

\section{Results}

The initial key word search for wrist ultrasound identified 272 subjects in whom a complete wrist extremity ultrasound examination was performed from January 2016 to April 2016. Of this group of subjects, $36 \%$ (98 of 272) had a wrist ganglion cyst on sonography, where $78 \%$ ( 76 of 98 ) were female and $22 \%$ (22 of 98) male subjects. The median age of all subjects was 51 years (range, 13-79 years). No subjects were excluded due to history of prior wrist surgery or treatment of a ganglion cyst.

In the 98 subjects in whom a wrist ganglion was identified on sonogram, a total of 124 ganglion cysts were present due to multiplicity of cysts in some subjects. Of these 124 ganglion cysts, $60 \%$ (75 of 124) were located volar and solitary, $22 \%$ (27 of 124$)$ were dorsal and solitary, and $18 \%$ (22 of 124$)$ were located both volar and dorsal in a unilateral wrist. Overall, $69 \%$ (86 of 124) of ganglion cysts were volar (Figures 1 and 2) and 31\% (38 of 124) were dorsal (Figures 3 and 4; Table 1). Of the above 98 subjects, 12 had bilateral ganglion cysts either volar (9 of 12), dorsal (1 of 12), or in both locations (2 of 12). With 
regard to volar wrist ganglion cysts, $81 \%$ (54 of 67) of subjects were female with a median age of 55 years (range, 13-70 years). With regard to dorsal wrist ganglion cysts, $71 \%$ (22 of 31 ) of subjects were female with a median age of 44 years (range, 17-74 years). Of the 124 wrist ganglion cysts, $2 \%$ (3 of 124) had surgical or histologic confirmation.

Of the 86 ganglion cysts located in the volar wrist, $91 \%$ ( 78 of 86 ) were located between the radial artery and the flexor carpi radialis tendon. The remaining $9 \%$ ( 8 of 86 ) volar wrist ganglia were located at the radiocarpal joint (4 of 86 ), at the pisiform ( 2 of 86$)$, at the capitate ( 1 of 86 ), and between the scaphoid and trapezium ( 1 of 86 ). Of the 38 ganglion cysts located in the dorsal wrist, $66 \%$ (25 of 38 ) were located superficial to the scapholunate ligament. Of the remaining $34 \%$ (13 of 38), $8 \%$ (3 of 38 ) were located at the first extensor compartment, 5\% (2 of 38 ) at the radiocarpal joint, $5 \%(2$ of 38$)$ at the

Figure 1. A 29-year-old woman with volar ganglion cyst. Color Doppler ultrasound images of volar wrist in (A) transverse and (B) sagittal planes show anechoic multilobular ganglion cyst (arrows) located between flexor carpi radialis (FCR) and radial artery (colored red in $\mathbf{A}$ ). Right side of image $\mathbf{B}$ is distal.
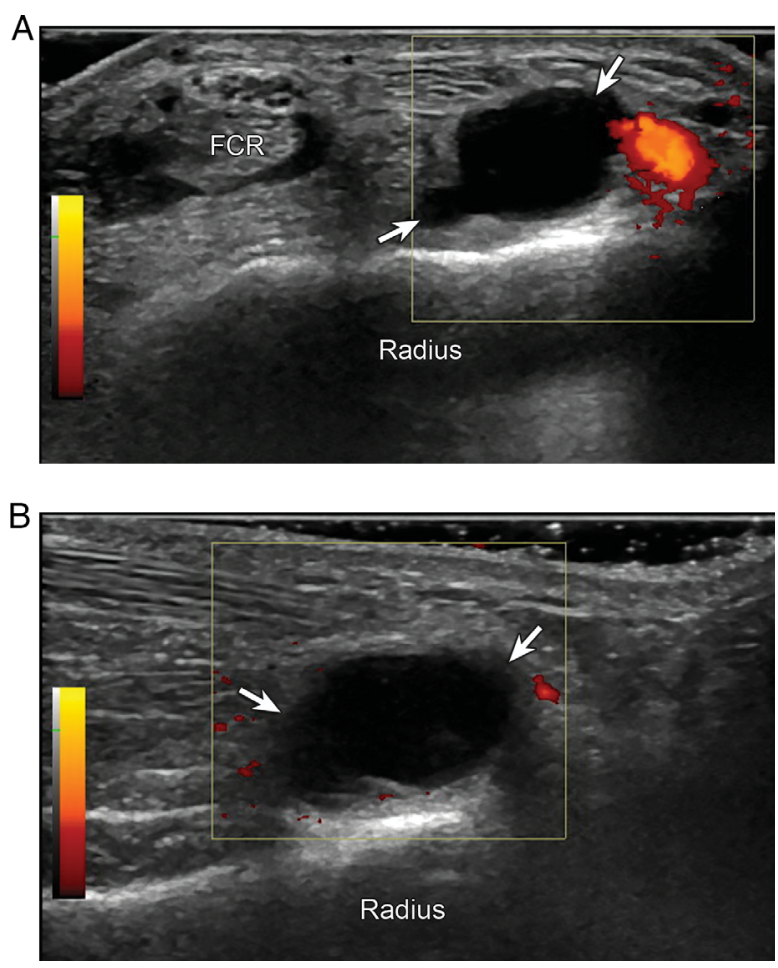

Figure 2. A 57-year-old woman with volar ganglion cyst. Ultrasound images of volar wrist in (A) transverse and (B) sagittal planes show hypoechoic multilobular ganglion cyst (arrows in $\mathbf{A}$ between cursors in $\mathbf{B}$ ) located between flexor carpi radialis (FCR) and radial artery (colored blue in $\mathbf{A}$ ). Right side of image $\mathbf{B}$ is distal.
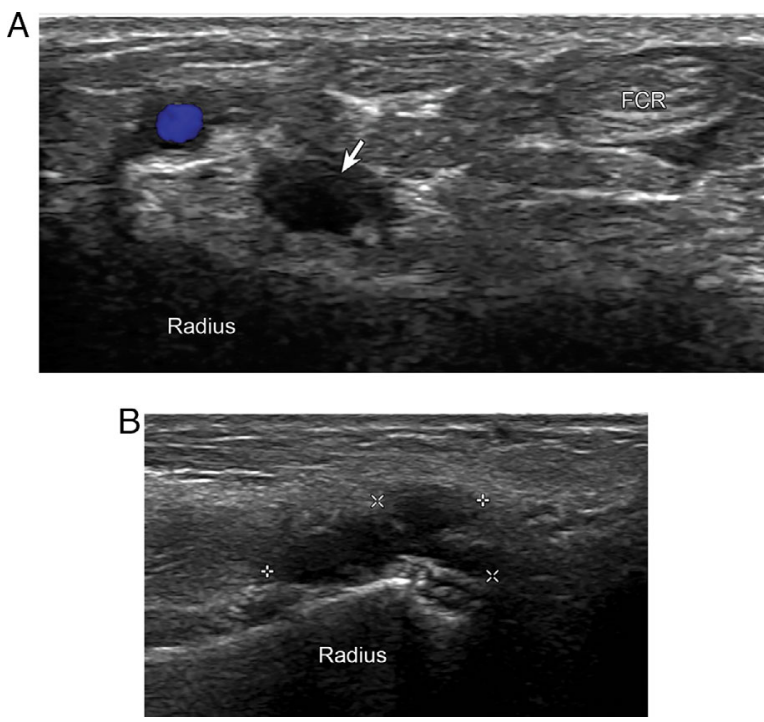

Figure 3. A 36-year-old woman with dorsal ganglion cyst. Ultrasound images of dorsal wrist in (A) sagittal and (B) transverse planes show anechoic multilobular ganglion cyst (between cursors). Right side of image $\mathbf{A}$ is distal.
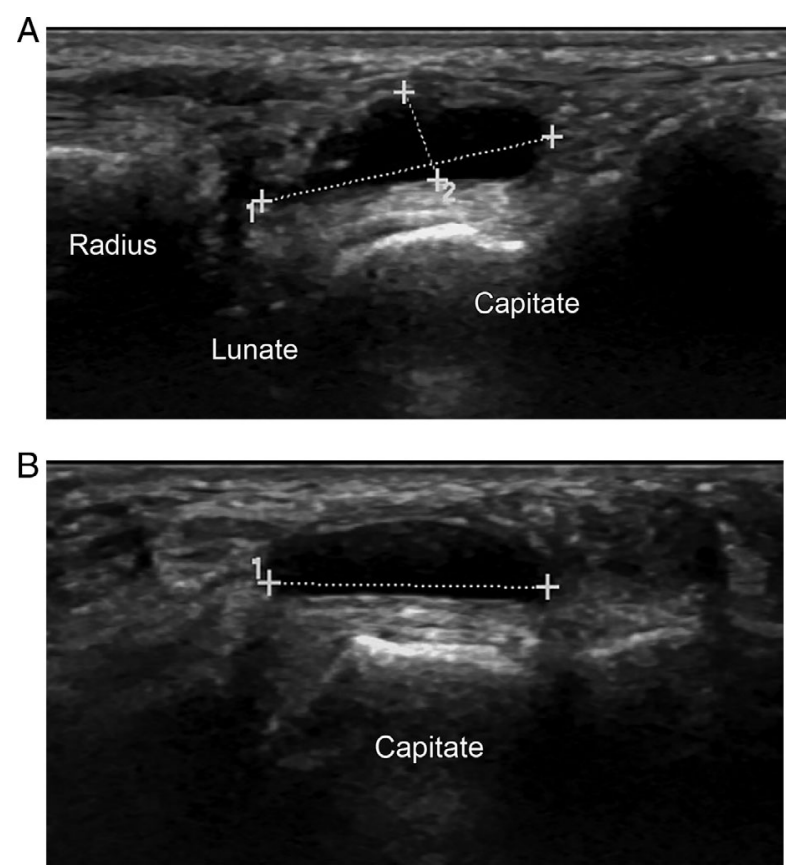
extensor carpi radialis tendons, $5 \%(2$ of 38$)$ at the fourth extensor compartment, 5\% (2 of 38) between the fourth and fifth extensor compartments, 3\% (1 of 38 ) at the extensor carpi ulnaris tendon near the triangular fibrocartilage complex, and $3 \%(1$ of 38 ) at the first carpometacarpal joint.

The mean largest dimension of the ganglion cysts was $1.0 \mathrm{~cm}$ (range, $0.2-2.5 \mathrm{~cm}$ ). All of the ganglion cysts (124 of 124) were multilocular or multilobular and none ( 0 of 124) were unilocular. Of the 22 dorsal ganglion cysts that were adjacent to the scapholunate ligament, 91\% (20 of 22) were associated with a normal-appearing scapholunate ligament on sonography (Figure 4).

Figure 4. A 37-year-old man with dorsal ganglion cyst. Ultrasound images of dorsal wrist in (A) sagittal and (B) transverse planes show anechoic multilobular ganglion cyst (between cursors). Note normal hyperechoic and fibrillar dorsal component of scapholunate ligament (arrowhead in B). Right side of image $\mathbf{A}$ is distal.
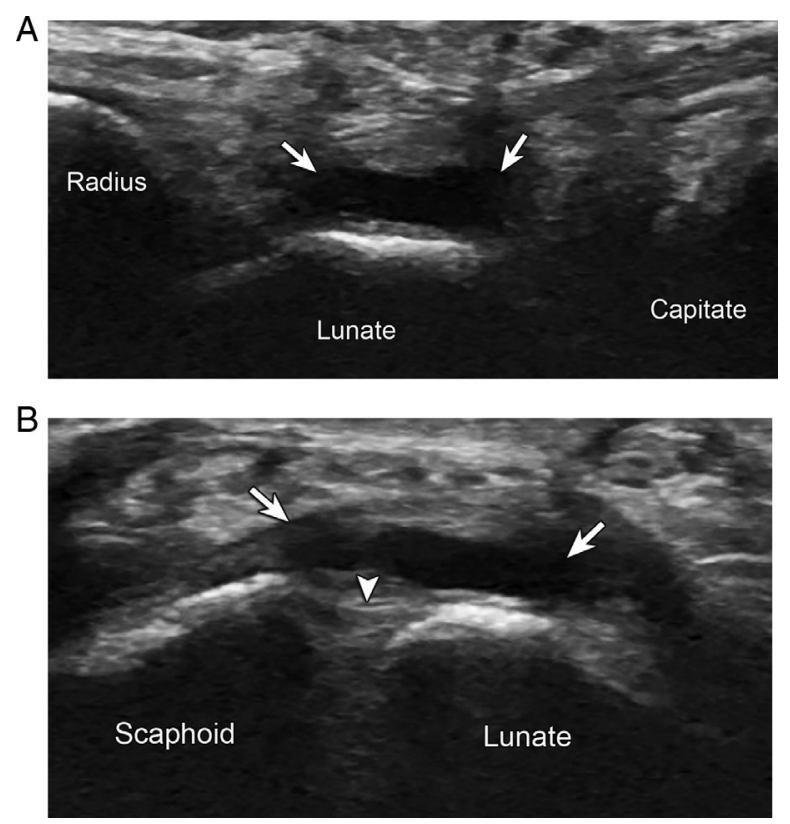

Table 1. Locations of Wrist Ganglion Cysts

\begin{tabular}{lccc}
\hline $\begin{array}{l}\text { Volar: } \\
\text { Radial Artery } \\
\text { and FCR }\end{array}$ & $\begin{array}{c}\text { Volar: } \\
\text { Other Sites }\end{array}$ & $\begin{array}{c}\text { Dorsal: } \\
\text { Scapholunate } \\
\text { Ligament }\end{array}$ & $\begin{array}{c}\text { Dorsal: } \\
\text { Other Sites }\end{array}$ \\
\hline $63 \%(78$ of 124$)$ & $6 \%(8$ of 124$)$ & $20 \%(25$ of 124$)$ & $11 \%(13$ of 124$)$ \\
\hline
\end{tabular}

FCR, flexor carpi radialis.

\section{Discussion}

In our patient population, $36 \%$ of all wrist ultrasound examinations showed a ganglion cyst. The most common location for wrist ganglion cysts was the volar wrist located between the radial artery and flexor carpi radialis (63\%), followed by a dorsal location superficial to the scapholunate ligament $(20 \%)$, other dorsal locations (11\%), and other volar locations (6\%). Ganglion cysts were multilocular or multilobular in all subjects.

Ganglion cysts are soft tissue myxoid masses with a fibrous capsule and no synovial lining. ${ }^{2}$ Their exact etiology is uncertain, but they are thought to be related to trauma or degenerative change, and they often have a connection to a nearby joint recess or tendon sheath. One popular theory is that ganglion cysts are created when fluid from these spaces is forced into surrounding tissues. ${ }^{2}$ Indeed, a communicating neck or pedicle between dorsal wrist ganglion cysts and the dorsal radiocarpal joint recess, and between a volar wrist ganglion cyst and the volar radiocarpal joint recess, has been described. ${ }^{10}$ While not specifically assessed in our retrospective study, similar connections between ganglion cyst and joint recess were identified. The propensity for ganglion cysts to occur in such specific anatomic locations may relate to sites of greatest mechanical stress or capsular weakening; the theory that injury is a prerequisite to ganglion cyst formation has been refuted. ${ }^{3}$ Indeed, specific locations of ganglion cysts reported in the literature include between the radial artery and flexor carpi radialis tendon, and superficial to the scapholunate interosseous ligament, which is similar to our study, in which $83 \%$ (103 of 124) were in these 2 locations. ${ }^{2}$

Evaluating more specific locations of ganglion cysts, the prior literature states that the majority $(60 \%-70 \%)$ of wrist ganglion cysts occur in the dorsal wrist. ${ }^{1-3,5,7}$ Contrary to the literature, our study has demonstrated ganglion cysts to be more common in a volar location (69\%). Most of the current literature cite older research regarding the most common locations of wrist ganglion cysts; much of this prior literature is based on clinical examination findings, where dorsal wrist ganglion cysts may be more readily identified. Surgical data on the most common locations of wrist ganglia are also likely to be heavily 
influenced by the clinical examination. The comprehensive ultrasound examination of the wrist in our subjects allowed a complete assessment for volar and dorsal ganglion cysts not biased by physical examination findings.

The high prevalence of wrist ganglion cysts in a volar location deserves further comment. While it is not known if the volar wrist ganglion cysts in this study specifically were symptomatic, causes of wrist pain may be multifactorial and symptoms may be referred. In addition, it has been shown that clinically occult wrist ganglion cysts may be a cause of wrist symptoms ${ }^{11}$; therefore, evaluation of the volar wrist between the radial artery and flexor carpi radialis by sonography or magnetic resonance imaging should be considered in patients with wrist symptoms given the propensity of ganglion cysts to occur at this location. One study using aspiration or surgery as the standard of reference showed that the majority of such treated ganglion cysts were indeed volar in location, which is further evidence of the potential clinical significance of volar ganglion cysts.

In our study, wrist ganglion cysts appeared hypoechoic, and in all cases the cysts were multilocular or multilobular. This may be explained by the proposed etiology of ganglion cysts where joint fluid is forced out through the joint capsule and subsequently dissects through tissue planes rather than distending a single-compartment anatomic space, such as a joint recess or bursa. This also explains why ganglion cysts are typically noncompressible, unlike a joint recess or bursa. 11

The dorsal wrist ganglion cysts in our study were most commonly located superficial to the scapholunate ligament, similar to prior studies. While it has been reported that such dorsal wrist ganglion cysts are due to degeneration of the scapholunate ligament, in $91 \%$ of our subjects the dorsal component of the scapholunate ligament had a normal hyperechoic and fibrillar appearance. This further supports that the location of such dorsal wrist ganglion cysts may not be related to a scapholunate ligament tear but rather distal extension of a ganglion cyst originating from the adjacent dorsal recess and joint capsule of the radiocarpal joint. ${ }^{3,10}$ A communicating neck or pedicle between the radiocarpal joint and dorsal ganglion cysts has been described in the literature and was seen in our cases. ${ }^{3,10}$
One limitation of our study was that ultrasound examinations were performed at only one institution, and validation of our results in a larger patient population would be important; however, our institution is a tertiary academic center with a large referral base and likely approximates the general population. Another limitation was that surgical or histologic confirmation was only present in $2 \%$, although established sonographic criteria for ganglion cysts were used for diagnosis. As an additional limitation, selection bias may be present, as symptomatic patients are more likely to seek medical attention; however, every wrist was comprehensively evaluated with sonography regardless of symptom location or clinical history. Regarding the integrity of the scapholunate ligament, only the dorsal aspect was assessed with sonography. Finally, the clinical significance of the ganglion cysts identified in this study was not assessed.

In conclusion, the most common location for wrist ganglion cysts is in the volar wrist, particularly between the radial artery and flexor carpi radialis tendon, appearing multilocular or multilobular with a mean largest dimension of $1.0 \mathrm{~cm}$. Given the propensity of ganglion cysts to occur at this site, attention to this area when imaging the wrist with sonography or magnetic resonance imaging should be considered.

\section{References}

1. Nguyen V, Choi J, Davis KW. Imaging of wrist masses. Curr Probl Diagn Radiol 2004; 33:147-160.

2. Thornburg LE. Ganglions of the hand and wrist. J Am Acad Orthop Surg 1999; 7:231-238.

3. Gude W, Morelli V. Ganglion cysts of the wrist: pathophysiology, clinical picture, and management. Curr Rev Musculoskelet Med 2008; 1:205-211.

4. Zeidenberg J, Aronowitz JG, Landy DC, Owens PW, Jose J. Ultrasound-guided aspiration of wrist ganglions: a follow-up survey of patient satisfaction and outcomes. Acta Radiol 2016; 57:481-486.

5. Teefey SA, Dahiya N, Middleton WD, Gelberman RH, Boyer MI. Ganglia of the hand and wrist: a sonographic analysis. AJR Am J Roentgenol 2008; 191:716-720.

6. Wang G, Jacobson JA, Feng FY, Girish G, Caoili EM, Brandon C. Sonography of wrist ganglion cysts: variable and noncystic appearances. J Ultrasound Med 2007; 26:1323-1328; quiz 1330-1321.

7. Meena S, Gupta A. Dorsal wrist ganglion: current review of literature. J Clin Orthop Trauma 2014; 5:59-64. 
8. Bianchi S, Abdelwahab IF, Zwass A, Giacomello P. Ultrasonographic evaluation of wrist ganglia. Skeletal Radiol 1994; 23:201-203.

9. Jacobson JA, Oh E, Propeck T, Jebson PJ, Jamadar DA, Hayes CW. Sonography of the scapholunate ligament in four cadaveric wrists: correlation with MR arthrography and anatomy. AJR Am J Roentgenol 2002; 179:523-527.
10. Freire V, Guerini H, Campagna R, et al. Imaging of hand and wrist cysts: a clinical approach. AJR Am J Roentgenol 2012; 199: W618-W628.

11. Cardinal E, Buckwalter KA, Braunstein EM, Mih AD. Occult dorsal carpal ganglion: comparison of US and MR imaging. Radiology 1994; 193:259-262. 